\title{
Quantitative Structural Analysis of Binary Nanocrystal Superlattices by Electron Tomography
}

\author{
Heiner Friedrich, ${ }^{*, \dagger}$ Cedric J. Gommes, ${ }^{\ddagger}$ Karin Overgaag, $, \$, \#$ Johannes D. Meeldijk," \\ Wiel H. Evers, ${ }^{\S}$ Bart de Nijs, ${ }^{\S}$ Mark P. Boneschanscher, ${ }^{\S}$ Petra E. de Jongh, ${ }^{\dagger}$ \\ Arie J. Verkleij," Krijn P. de Jong, ${ }^{\dagger}$ Alfons van Blaaderen, ${ }^{\perp}$ \\ and Daniel Vanmaekelbergh ${ }^{\star, \S}$ \\ Inorganic Chemistry and Catalysis, Debye Institute for Nanomaterials Science, Utrecht \\ University, Sorbonnelaan 16, Utrecht 3584 CA, The Netherlands, Department of \\ Chemical Engineering, University of Liège, Allée du 6 août 3, 4000 Liège, Belgium, \\ Condensed Matter and Interfaces, Soft Condensed Matter, Debye Institute for \\ Nanomaterials Science, Utrecht University, Princetonplein 1, Utrecht 3584 CC, \\ The Netherlands, and Electron Microscopy Group, Utrecht University, Padualaan 8, \\ Utrecht $3584 \mathrm{CH}$, The Netherlands
}

Received April 16, 2009; Revised Manuscript Received May 29, 2009

\begin{abstract}
Binary nanocrystal superlattices, that is, ordered structures of two sorts of nanocolloids, hold promise for a series of functional materials with novel collective properties. Here we show that based on electron tomography a comprehensive, quantitative, three-dimensional characterization of these systems down to the single nanocrystal level can be achieved, which is key in understanding the emerging materials properties. On four binary lattices composed of PbSe, CdSe, and Au nanocrystals, we illustrate that ambiguous interpretations based on two-dimensional transmission electron microscopy can be prevented, nanocrystal sizes and superlattice parameters accurately determined, individual crystallographic point and plane defects studied, and the order/disorder at the top and bottom surfaces imaged. Furthermore, our results suggest that superlattice nucleation and growth occurred at the suspension/air interface and that the unit cells of some lattices are anisotropically deformed upon drying.
\end{abstract}

Bottom-up assembly of prefabricated building blocks into large, ordered structures by lowering of the free energy is a concept shared across several disciplines from biology, supramolecular chemistry to the crystallization of microcolloids. Particles in the nanometer size range are of special interest here, as they bridge the worlds of molecular and microcolloidal self-organization. In addition, the nanometer dimension crosses a number of natural physical length scales resulting in properties that strongly depend on the nanocrystal (NC) size and shape. For instance, semiconductor NCs show a

\footnotetext{
* To whom correspondence should be addressed. E-mail: d.vanmaekelbergh@uu.nl and h.friedrich@uu.nl.

${ }^{\dagger}$ Inorganic Chemistry and Catalysis, Debye Institute for Nanomaterials Science, Utrecht University.

\# Department of Chemical Engineering, University of Liège.

${ }^{\S}$ Condensed Matter and Interfaces, Debye Institute for Nanomaterials Science, Utrecht University.

"Electron Microscopy Group, Utrecht University.

${ }^{\perp}$ Soft Condensed Matter, Debye Institute for Nanomaterials Science, Utrecht University.

\# Present address: Shell International E\&P, Postbus 80, 2280 AB Rijswijk,
} The Netherlands. discrete electronic structure and size-tunable opto-electrical properties on account of quantum confinement. ${ }^{1}$

Crystallization of nanocolloids has led to a plethora of single-component and, more recently, binary superlattices of striking structural diversity, long-range order and beauty. ${ }^{2-7}$ Particularly binary nanocrystal superlattices, that is, ordered structures of two sorts of nanocolloids that are metallic, insulating, semiconducting, or magnetic in nature, hold promise for a series of novel materials with thermo-, magneto- or opto-electric applications. ${ }^{7-9}$ The special potential of binary systems in which one type of $\mathrm{NC}$ is in close contact with another lies in the interactions between the building blocks that can induce novel collective properties. A striking example has been published recently. NCs that are inherently insulating, when assembled in a binary superlattice, led to a material with strongly enhanced conductivity. ${ }^{7}$ The material's properties will depend critically on the overall superlattice geometry, the distances between the NCs, and the density and nature of defects. Hence, understanding the emerging properties of such systems 
demands a full structural characterization of the superlattice in three dimensions (3D), including its defects. Moreover, such a structural analysis can also shed light on the formation mechanism of the superlattice and driving force(s) of colloid crystallization, information that is lacking at the moment

Techniques that analyze the spatial Fourier transform of a real space structure such as (small-angle) X-ray diffraction ${ }^{10}$ and electron crystallography ${ }^{11}$ have proven to elucidate the average crystal lattice accurately. However, a drawback of these methods is that local, spatially resolved information cannot be obtained, which makes them not suitable for the study of superlattice boundaries and defects. In contrast, electron tomography (ET), which has been employed in structural biology ${ }^{12,13}$ for many years and more recently in materials science, ${ }^{14-17}$ can provide local, spatially resolved information on the nanoscale and in 3D. Here, we show that ET can be extended to a comprehensive structural analysis of binary $\mathrm{NC}$ superlattices by quantifying individual $\mathrm{NC}$ coordinates, sizes, unit cell parameters, and furthermore characterizing point and plane defects.

Four binary superlattices of $\mathrm{AB}_{2}$ and $\mathrm{AB}_{13}$ stoichiometry ( $\mathrm{PbSe}$ and $\mathrm{CdSe} \mathrm{NCs}$ ) and of $\mathrm{AB}_{2}$ and $\mathrm{AB}$ stoichiometry (PbSe and $\mathrm{Au} \mathrm{NCs)} \mathrm{were} \mathrm{prepared} \mathrm{as} \mathrm{previously} \mathrm{published.}{ }^{5}$ In short, mixed suspensions of trioctylphosphine oxide (TOPO)/hexadecylamine (HDA) capped-CdSe and oleic acid (OA)-capped PbSe nanocrystals (NCs) were prepared in tetrachloroethylene (TCE), while suspensions of dodecanethiol-capped gold NCs and OA-capped PbSe NCs were prepared in toluene in an oxygen and water-free environment. By varying the $\mathrm{NC}$ concentrations in suspension different binary superlattices could be prepared. Colloidal crystallization proceeded by evaporating the solvent under reduced pressure ( $\sim 10$ mbar) at $70{ }^{\circ} \mathrm{C}$ with the substrate kept at an angle of $30^{\circ}$ to the horizontal. Carbon-coated Formvar films supported on $\mathrm{Cu}$ TEM grids were used as substrates. $\mathrm{PbSe} /$ CdSe NC structures of $\mathrm{AB}_{2}$ and $\mathrm{AB}_{13}$ stoichiometry were mainly formed at a $\mathrm{c}_{\mathrm{CdSe}} / \mathrm{c}_{\mathrm{PbSe}}$ of 5 and 12 , respectively. $\mathrm{PbSe} /$ $\mathrm{Au}$ structures of $\mathrm{AB}$ and $\mathrm{AB}_{2}$ stoichiometry were formed at $\mathrm{a} \mathrm{c}_{\mathrm{Au}} / \mathrm{c}_{\mathrm{PbSe}}$ ratio between 1 and 5 . The particle concentration ratios were determined from absorption measurements using a Lambda 950 UVvis spectrometer.

Electron tomography was performed in bright-field mode using a Tecnai 20 electron microscope with a $\mathrm{LaB}_{6}$ electron source (FEI Company, Eindhoven). Images of three tilt-series were acquired over an angular range of $\pm 70^{\circ}$ at $2^{\circ}$ increments on a Tietz $2 \mathrm{k} \times 2 \mathrm{k}$ charge-coupled device camera (TVIPS Gmbh, Gauting) using Inspect3D software (FEI Company, Eindhoven). The nominal magnification was $29000 \times$ corresponding to a pixel size of $0.27 \mathrm{~nm}$. The defocus was set to -200 to $-300 \mathrm{~nm}$ (nominal) to keep the entire object in underfocus throughout the tilt-series and an objective aperture of $40 \mu \mathrm{m}$ was inserted in the beam path. Images of the tilt-series were aligned with respect to a common origin and rotation axis using the $\mathrm{PbSe}$ or $\mathrm{Au}$ particles as fiducial markers. The aligned tilt-series was binned to $0.54 \mathrm{~nm}$ pixel size, which is approximately the interpretable resolution at the experimental imaging conditions prior to reconstruction of the three-dimensional (3D) volume. An example is provided in the Supporting Information (SImovie1.mpg) showing an aligned tilt series of the $\mathrm{AB}_{13}$ lattice acquired over an angular range of $\pm 70^{\circ}$ at $2^{\circ}$ increments. This data acquisition scheme is commonly referred to as single-axis tilting. ${ }^{18}$ The $3 \mathrm{D}$ intensity map of the object is reconstructed by weighted backprojection. ${ }^{19}$ The corresponding reconstruction of the $\mathrm{AB}_{13}$ lattice is displayed in Supporting Information, SImovie2.mpg by sequential numerical cross sections parallel to the imaging plane at different heights (see also cartoon in Figure 1a). SImovie2 starts at the back side of the support film, moving upward through the support film and the superlattice and then back downward. A comprehensive description of electron tomography can be found in refs 14, 15, 18, and 19. Alignment and reconstruction by weighted backprojection was performed in IMOD. ${ }^{20}$

Image analysis in Matlab determined NC coordinates by template matching ${ }^{21,22}$ and $\mathrm{NC}$ core diameters from the radial intensity profiles. ${ }^{23}$ This enabled us to characterize thousands of $\mathrm{NC}$ in each tomogram containing less than $5 \%$ false positives for high-contrast $\mathrm{PbSe}$ and $\mathrm{Au} \mathrm{NCs}$ and around $10 \%$ false positives for the low-contrast CdSe NCs. We estimate that, overall, approximately $90 \%$ of the NCs were detected. Subsequently, positional order, but also local symmetries as revealed by local bond-order parameters, ${ }^{24-26}$ were exploited to find individual crystallites. Furthermore, we fitted lattice vectors, determined unit cell positions and neighbor distances in 4-5 subregions of the tomograms each containing a few hundred NCs. More details on individual steps of the analysis and examined subregions are provided in the Supporting Information.

The crystallographic analysis of the $\mathrm{AB}_{2}$ superlattice containing $\mathrm{PbSe}$ and $\mathrm{CdSe}$ NCs is summarized in Figure 1b-e (see Supporting Information, Figure SI 1a and SI Table 1 for more details). The lattice parameters varied less than $5 \%$ between different subregions, NC core diameters were constant (9 $\mathrm{nm} \mathrm{PbSe} ; 3.4 \mathrm{~nm} \mathrm{CdSe}$ ), and a thickness of 4 to 5 unit cells was observed. The relative positions of the NCs in the unit cell confirmed ordering isostructural with $\mathrm{AlB}_{2}$ (space group 191), however, with a strong contraction perpendicular to the substrate plane (C-vector). While in the [001] plane (Figure 1c) the PbSe NC are well separated by the organic ligand shell, in the [1-10] plane (Figure 1d) no gap between the NC cores is visible. The absence of a visible gap in the [1-10] plane results from a compression of the organic ligand shell perpendicular to the support and from blurring by the reconstruction point spread function. The reason for the strong anisotropic contraction will be discussed below in context of superlattice formation. More details on the second $\mathrm{AB}_{2}$ lattice $(7.1 \mathrm{~nm} \mathrm{PbSe} ; 3.7 \mathrm{~nm} \mathrm{Au}$; thickness 2-4 unit cells) also exposing $\mathrm{AlB}_{2}$ ordering and contraction are provided in Supporting Information, Figure SI 1b, Figure SI 2, and SI Table 2.

Furthermore, we investigated lattices with $\mathrm{AB}_{13}$ stoichiometry (8.8 nm PbSe; $3.7 \mathrm{~nm} \mathrm{CdSe}$ ), which are most intriguing as two isomorphs exist. Previous TEM studies reported the occurrence of the cub-octahedral $\mathrm{AB}_{13}$ structure. ${ }^{5,6}$ Since this particular arrangement has a considerably 


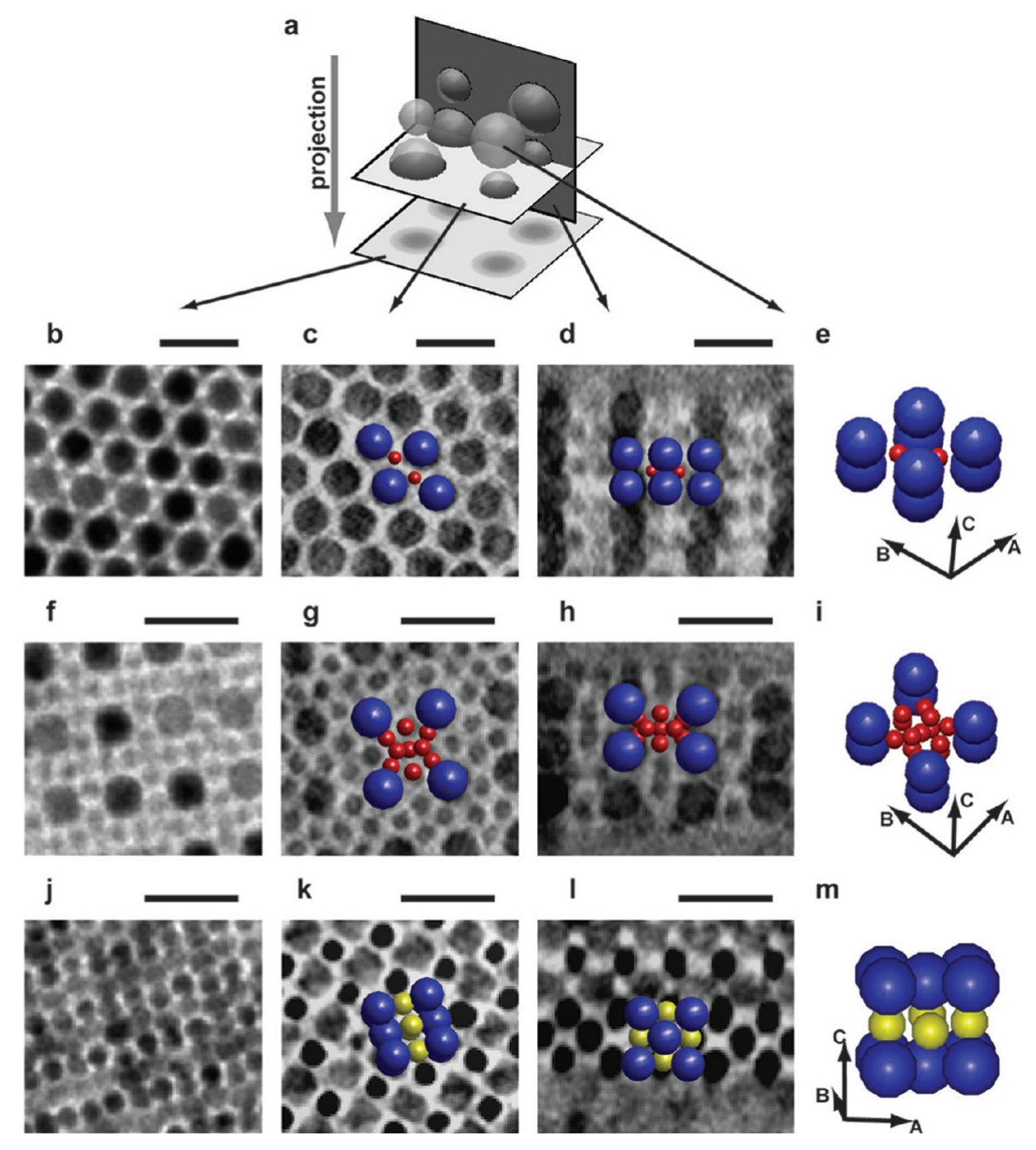

Figure 1. Crystallographic analysis of binary superlattices with $\mathrm{AB}_{2}, \mathrm{AB}_{13}$, and $\mathrm{AB}$ stoichiometry. (a) Cartoon explaining the relationship between the panels below. TEM image (left), numerical cross section through $3 \mathrm{D}$ reconstruction (3D intensity map) parallel to imaging plane (left center), numerical cross section perpendicular to imaging plane (right center), and unit cell showing the nanocrystal cores with lattice vectors (right). (b-e) $\mathrm{AB}_{2}$ superlattice of $9 \mathrm{~nm} \mathrm{PbSe}$ (blue) and $3.4 \mathrm{~nm}$ CdSe NCs (red) with (b) TEM image interpolated from 0.54 $\mathrm{nm} / \mathrm{px}$ along (001) direction at zero tilt, (c) cross section through [001] plane of PbSe NCs, the CdSe NCs are 1/2 unit cell higher, (d) cross section through [1-10] plane of PbSe and CdSe NCs, and (e) $\mathrm{AB}_{2}$ unit cell. (f-i) ico- $\mathrm{AB}_{13}$ superlattice of $8.8 \mathrm{~nm} \mathrm{PbSe}(\mathrm{blue})$ and $3.8 \mathrm{~nm}$ CdSe (red) NCs (1/8th unit cell) with (f) TEM image interpolated from $0.54 \mathrm{~nm} / \mathrm{px}$ along (001) direction at zero tilt, (g) cross section parallel to [001] plane through $\mathrm{PbSe}$ (dark gray) and four nearest CdSe (light gray), (h) cross section parallel to [010] plane through PbSe and two nearest CdSe, and (i) 1/8th of ico- $\mathrm{AB}_{13}$ unit cell. $(\mathrm{j}-\mathrm{m}) \mathrm{AB}$ superlattice of $6.8 \mathrm{~nm} \mathrm{PbSe}$ (blue) and $4.6 \mathrm{~nm}$ Au (yellow) NCs with (j) TEM image interpolated from $0.54 \mathrm{~nm} / \mathrm{px}$ along (010) direction at zero tilt, (k) cross section through [010] plane of PbSe (dark gray) and Au (black), (1) cross section through [001] plane of PbSe and Au NCs, (m) and AB unit cell. Scale bars are $20 \mathrm{~nm}$.

lower filling fraction than the icosahedral $\mathrm{AB}_{13}$ isomorph, the suitability of the hard-sphere model $^{27}$ for the studied systems has been put into question. As in the previous reports, the TEM image (Figure 1f) may equally give the impression that the structure is cub-octahedral. In contrast, the numerical cross section in Figure $1 \mathrm{~g}$ reveals that the four CdSe closest to the [001] lattice plane do not form a square. Instead, the CdSe quadrangle is elongated in one direction, alternating by $90^{\circ}$ degrees between neighboring cells. The numerical cross section in Figure $1 \mathrm{~h}$ slices through the $\mathrm{PbSe}$ and the two CdSe closest to the [010] lattice plane. The relative location of the $\mathrm{CdSe} \mathrm{NCs}$ alternates by $90^{\circ}$ between neighboring cells, as the $\mathrm{CdSe}$ are either being visible side by side or above each other. The observation of a $90^{\circ}$ rotation in the CdSe arrangement between neighboring cells reveals without any doubt the icosahedral configuration. ET analysis on all other subregions (Supporting Information, Figure SI 3) shows that the densest $A_{13}$ structure, that is, the icosahedral isomorph, prevails. A flawed analysis based on TEM images may arise from the complexity of the ico- $\mathrm{AB}_{13}$ lattice with a layer-wise rotation of the CdSe "cross motif" along the projection axis. The structure depicted in Figure 1f is one ico- $\mathrm{AB}_{13}$ unit cell thick (Figure 1h), which corresponds to the projection of two by $90^{\circ}$ rotated cells. Analogous to the $A_{2}$ examples, the $A_{13}$ lattices are strongly contracted perpendicular to the support resulting in a distance of 1-2 $\mathrm{nm}$ between the PbSe $\mathrm{NC}$ cores in this direction. More details are provided in Supporting Information, Figure SI 1c and SI Table 3.

The last example presented in Figure $1 \mathrm{j}-\mathrm{m}$ summarizes the crystallographic analysis of an $\mathrm{AB}$ superlattice formed by $6.9 \mathrm{~nm} \mathrm{PbSe}$ and $4.6 \mathrm{~nm}$ Au NCs. Again, the TEM image 
a

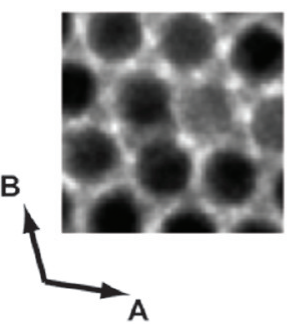

d

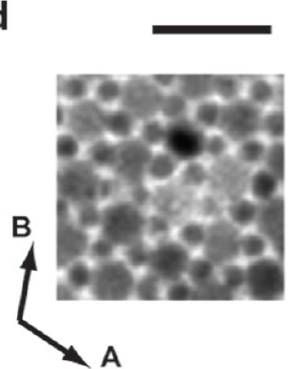

g

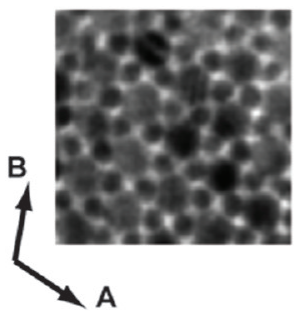

j

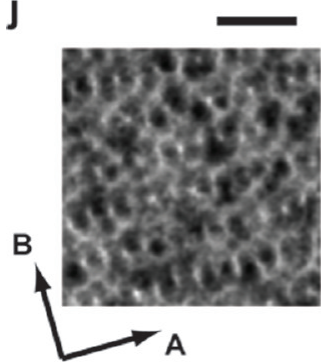

b

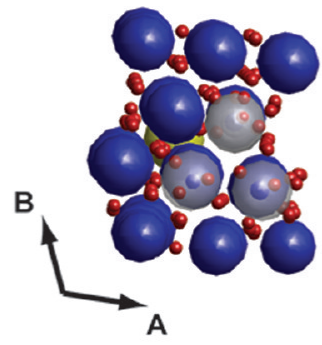

e

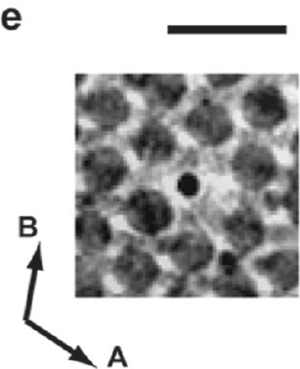

h

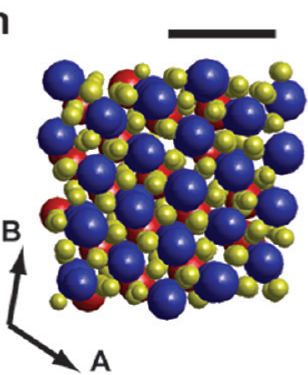

k

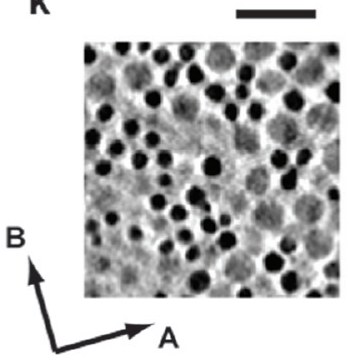

C

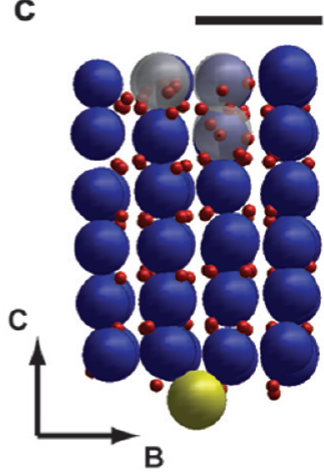

f
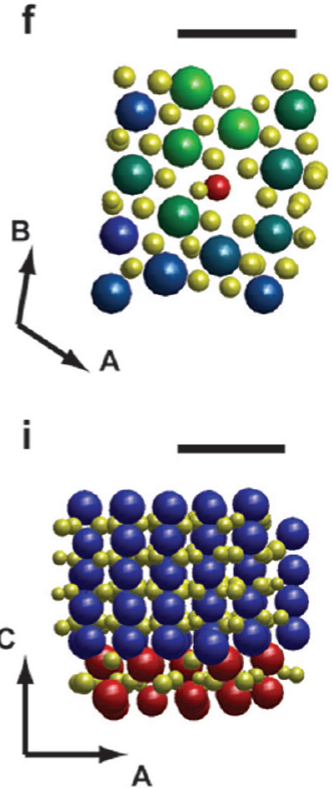

I

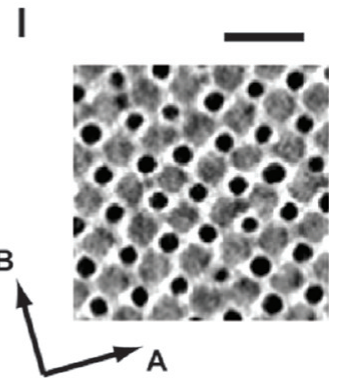

Figure 2. Examples of defects in binary superlattices with $\mathrm{AB}_{2}$, and $\mathrm{AB}$ stoichiometry. Corresponding panels show TEM image interpolated from $0.54 \mathrm{~nm} / \mathrm{px}$ (left) and ET analysis (center and right) of same region. (a) TEM image $\mathrm{AB}_{2}$ of $\mathrm{PbSe}$ and $\mathrm{CdSe}$ along (001) direction at zero tilt, $(\mathrm{b}, \mathrm{c}) \mathrm{ET}$ analysis with $\mathrm{PbSe}$ in blue, $\mathrm{CdSe}$ in red, substituted $\mathrm{PbSe}$ in semitransparent white, and surface adsorbed PbSe in yellow. (d) TEM image $\mathrm{AB}_{2}$ of $\mathrm{PbSe}$ and $\mathrm{Au}$ along (001) direction at zero tilt, (e) numerical cross section through reconstruction parallel to imaging plane with Au substitution in black and stretched PbSe lattice in dark gray, (f) ET analysis with PbSe color coded relative to displacement from lattice (blue $=$ on lattice, green $=2.5 \mathrm{~nm}$ of lattice), Au on lattice in yellow and PbSe substitution in red. (g) TEM image $\mathrm{AB}_{2}$ of $\mathrm{PbSe}$ and $\mathrm{Au}$ along (001) direction at zero tilt, (h,i) ET analysis with PbSe in blue, red and $\mathrm{Au}$ in yellow showing grain boundary parallel to support. (j) TEM image $\mathrm{AB}$ of PbSe and Au along (010) direction at zero tilt, (k) numerical cross section parallel to the imaging plane through first layer from support, (1) second layer from support with Au in black and PbSe in dark gray. Scale bars are $20 \mathrm{~nm}$.

(Figure 1j) is complex and prone to misinterpretation. The numerical cross sections parallel (Figure 1k, [010] plane) and perpendicular (Figure 11, [001] plane) to the support film illustrate that a superlattice isostructural with $\mathrm{CuAu}$ (space group 123) is present. Most notably, the stacking perpendicular to the support film contains a 90 degree rotation between the first and second unit cell layer (Figure 11), responsible for the complex TEM projection pattern. The examined $\mathrm{AB}$ superlattice was 2 to 3 unit cells thick. The lattice parameters did not vary by more than $4 \%$ between different regions. Interestingly, we find no contraction perpendicular to the support film as for the superlattices discussed above (see Supporting Information, Figure SI 1d and SI Table 4 for more details). 
In NC superlattices, as in atomic crystals, entropy dictates and gives a lower bound on the number and types of crystallographic defects, such as NC vacancies and substitutions. Kinetic effects during the superlattice growth can, however, significantly increase their number. In Figure 2 we illustrate that ET is, in contrast to 2D TEM and scattering methods, also extremely valuable to discern crystal defects. While the TEM images in Figure 2a,d,g suggest defect-free $\mathrm{AB}_{2}$ superlattices, ET analysis reveals that each region contains one or more crystallographic defects. For example, the $3 \mathrm{D}$ representations in Figure 2b,c show PbSe NC vacancies occupied by multiple $\mathrm{CdSe}$ NCs and a surfaceadsorbed $\mathrm{PbSe} \mathrm{NC}$ attached between the superlattice and the support film. Likewise, a numerical cross section through an $\mathrm{AB}_{2}$ structure composed of $\mathrm{PbSe}$ and $\mathrm{Au}$ NCs (Figure $2 \mathrm{e}$ ) and the corresponding $3 \mathrm{D}$ representation (Figure $2 \mathrm{f}$ ) clearly reveal a substitutional defect, almost invisible in the TEM image (Figure 2d) of the 3 unit cell thick specimen. Contrary to the fact that a $\mathrm{PbSe} \mathrm{NC}$ has been replaced by a smaller $\mathrm{Au} \mathrm{NC}$, the lattice appears to be stretched around the point defect. The magnitude of displacement for the $\mathrm{PbSe}$ NCs is represented in Figure $2 \mathrm{f}$ (and in Supporting Information, Figure SI 4 for a larger region) as a color change from blue (on lattice) to green ( $\sim 2.5 \mathrm{~nm}$ of lattice). One can envision that developments toward strain field mapping could bridge the gap between the $3 \mathrm{D}$ mico-colloidal ${ }^{25}$ and $2 \mathrm{D}$ atomic $^{28}$ equivalent. Furthermore, quantifying defect densities by visual inspection (e.g., 3.6\% Au NC vacancies were found in the $\mathrm{AB}_{2}$ lattice), or even better by automated routines, seems an obvious extension for the future.

Besides point defects, plane defects and order/disorder transitions could also be inferred by ET. For example, Figure $2 \mathrm{~h}, \mathrm{i}$ displays $3 \mathrm{D}$ representations of a grain boundary parallel to the support surface, characterized by a horizontal shift by half a unit cell. Likewise, in AB lattices the above-mentioned rotation of the unit cell over $90^{\circ}$ from layer to layer (Figure 11) was detected. Moreover, NCs randomly adsorbed on the top or bottom surface can significantly obstruct TEM imaging of an underlying lattice. This situation is exemplified in Figure $2 \mathrm{j}$, presenting a TEM image that suggests a disordered NC film. ET slices reveal, however, that this is due to one layer of surface adsorbed NCs (Figure 2k) on the bottom surface of a defect-free AB superlattice (Figure 2l). Alternatively, electron diffraction or analysis of the TEM image by fast Fourier transform (Supporting Information, Figure SI 5) will expose the underlying lattice periodicity but not the disordered layer as shown in Figure 2k. Additional examples of crystallographic defects can be found in Supporting Information, Figure SI 6.

Furthermore, being able to study the top and bottom surfaces by ET can give important information regarding the nucleation and growth mechanism. Figure 3 shows a TEM image of the $\mathrm{AB}_{13}$ superlattice together with three numerical cross sections perpendicular to the support film. The top of each cross section (Figure $3 b-d$ ) corresponds to the suspension/air interface, the bottom to the suspension/support boundary. It can be seen that the top region of the sections is mainly well ordered, while the bottom displays surface-

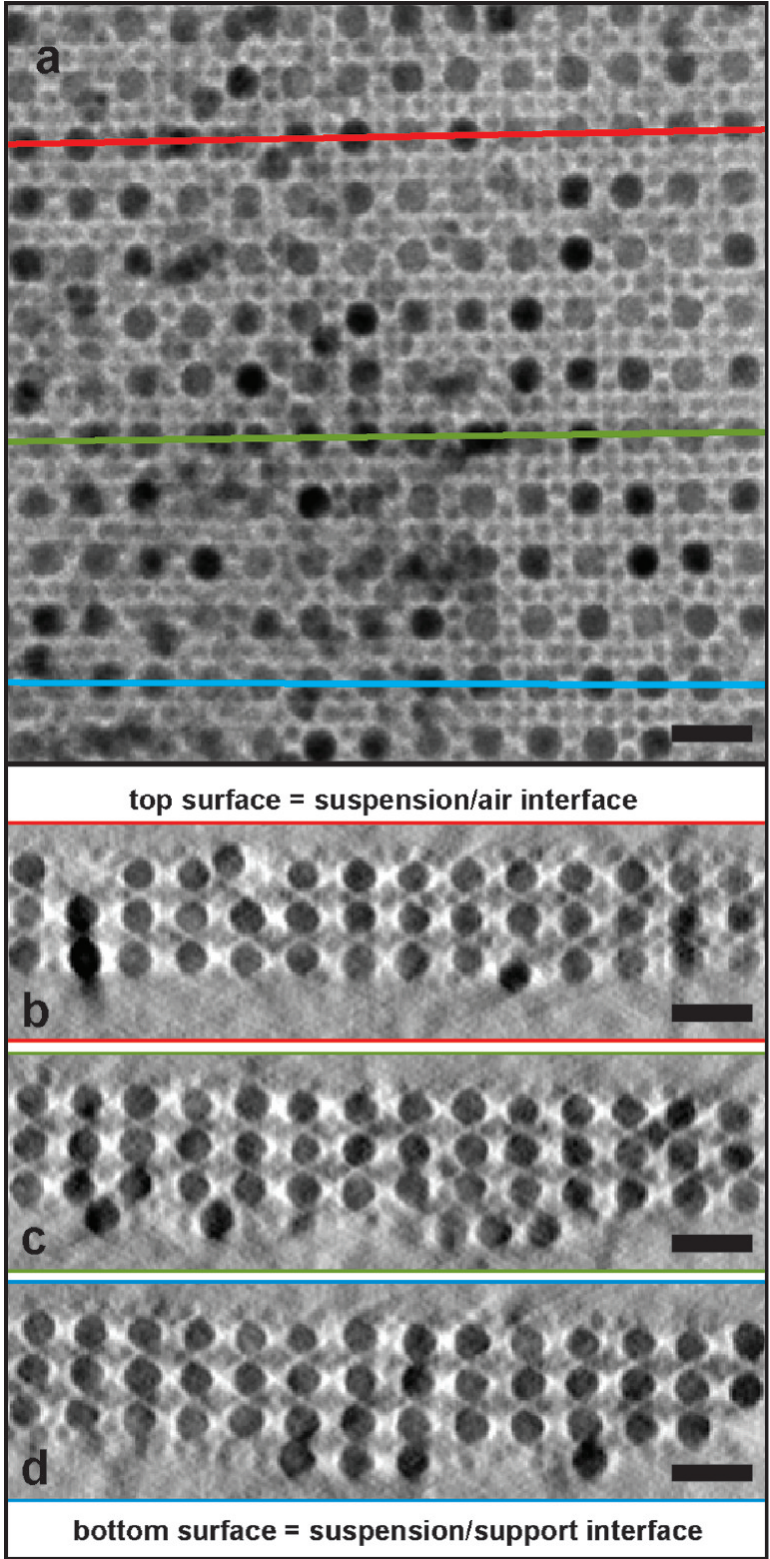

Figure 3. Structure of top and bottom surfaces in $\mathrm{AB}_{13}$ superlattice consisting of PbSe and CdSe NCs. (a) TEM image along (001) direction at zero tilt with positions of numerical cross sections perpendicular to the imaging plane indicated in red, green, and blue. $(b-d)$ Numerical cross sections perpendicular to support. The top surface, that is, the suspension/air interface, is well ordered, while the bottom surface, that is, the suspension/support interface, shows surface-adsorbed $\mathrm{PbSe}(\mathrm{c})$ and unfinished NC layers (d). Scale bars are $20 \mathrm{~nm}$.

adsorbed NCs (Figure 3c) and unfinished NC layers (Figure 3d). An unfinished layer means in this context that PbSe NCs are present in crystallographic positions in the fourth layer from the top while not all positions in the fourth layer are occupied by PbSe NCs. Similar observations can be made for the $\mathrm{AB}_{2}$ lattices (Supporting Information, Figure SI 7a-h) and the AB lattice (Supporting Information, Figure SI 71-o). Overall the top crystal layers are mainly well-ordered and continuous while step edges and disordered layers are found toward the bottom of the lattices. This provides strong evidence that the nucleation of the binary NC superlattices was not initiated at the support/suspension interface. A very 
recent study concluded that superlattices crystallize at the suspension/air interface and then deposit as a floating interfacial layer onto the substrate..$^{29}$ Reports of experiments with casted drops of $\mathrm{NC}$ suspensions support this notion. ${ }^{30}$ Likewise, microcolloid crystallization induced by evaporating the solvent can also be initiated at the solvent/air interface. ${ }^{31}$

The superlattice most likely has grown laterally along the interface and downward during further evaporation of the solvent. Superlattice growth becomes "confined" when the bottom surface gets close to the support film. In this view, the bottom lines of the perpendicular intersections provide indications of the superlattice growth close to a wall when the NCs were depleted. The contraction of the superlattice in the direction perpendicular to the support film is then tentatively explained by an anisotropic response to forces of the evaporating solvent retracting through the superlattice during drying. ${ }^{29,32}$ Regarding the possible use of NC superlattices, we anticipate that, for example, anisotropic lattice contractions and layer-wise rotations of the unit cell must have a dramatic effect on charge carrier generation and transport.

In summary, we have shown that electron tomography and image analysis can achieve single NC detection and, thus, is an indispensable method for a quantitative in depth real space study of (binary) nanocrystal superlattices. ET provides accurate lattice parameters of a given superlattice, unambiguously revealing the crystal structure including possible distortions. Furthermore, the ability to detect defects and their effect on the surrounding lattice will be instrumental in a microscopic understanding of emerging materials properties. ${ }^{7-9}$ In addition, real space analysis already has given clues to crystal nucleation and growth mechanisms, which can be improved by arresting these processes via, for example, a fast temperature quench. Moreover, we anticipate that, as in the microcolloid equivalent where quantitative 3D optical microscopy on a single particle level has led to new insights into fundamental problems such as the glass transition, ${ }^{33-35}$ crystallization ${ }^{25}$ and finally to a determination of interparticle potentials, ${ }^{36}$ electron tomography will be instrumental in providing similar insights on the nanoscale.

Acknowledgment. H.F. and K.P.dJ. acknowledge financial support from The Netherlands Research School Combination Catalysis (NRSC-C). C.J.G. is a postdoctoral researcher of F.R.S.-FNRS (Belgium).

Supporting Information Available: Media files showing an aligned tilt-series (SImovie1.mpg) and the corresponding $3 \mathrm{D}$ reconstruction (SImovie2.mpg) of the $\mathrm{AB}_{13}$ lattice and a text file containing details of the image and structure analysis, TEM images of examined superlattices and analyzed subregions, crystallographic analysis of a second $A_{2}$ superlattice, numerical cross sections illustrating the prevailing ico$\mathrm{AB}_{13}$ configuration, displacement analysis of $\mathrm{AB}_{2}$ superlattice, illustration of underlying symmetry in $\mathrm{AB}$ superlattices by Fourier transform, examples of crystallographic defects in superlattices, analysis of the top and bottom surfaces in $A B$ and $\mathrm{AB}_{2}$ superlattices, and supplementary tables containing structural parameters of all examined superlattices. This material is available free of charge via the Internet at http:// pubs.acs.org.

\section{References}

(1) Weller, H. Adv. Mater. 1993, 5, 88-95.

(2) Kiely, C. J.; Fink, J.; Brust, M.; Bethell, D.; Schiffrin, D. J. Nature 1998, 396, 444-446.

(3) Rogach, A. L. Angew. Chem., Int Ed. 2004, 43, 148-149.

(4) Shevchenko, E. V.; Talapin, D. V.; Kotov, N. A.; O’Brien, S.; Murray, C. B. Nature 2006, 439, 55-59.

(5) Overgaag, K.; Evers, W.; de Nijs, B.; Koole, R.; Meeldijk, J.; Vanmaekelbergh, D. J. Am. Chem. Soc. 2008, 130, 7833-7835.

(6) Shevchenko, E. V.; Talapin, D. V.; O'Brien, S.; Murray, C. B. J. Am. Chem. Soc. 2005, 127, 8741-8747.

(7) Urban, J. J.; Talapin, D. V.; Shevchenko, E. V.; Kagan, C. R.; Murray, C. B. Nat. Mater. 2007, 6, 115-121.

(8) Gur, I.; Fromer, N. A.; Geier, M. L.; Alivisatos, A. P. Science 2005, 310, 462-465.

(9) Talapin, D. V.; Murray, C. B. Science 2005, 310, 86-89.

(10) Connolly, S.; Fullam, S.; Korgel, B.; Fitzmaurice, D. J. Am. Chem. Soc. 1998, 120, 2969-2970.

(11) Sakamoto, Y.; Kaneda, M.; Terasaki, O.; Zhao, D. Y.; Kim, J. M.; Stucky, G.; Shim, H. J.; Ryoo, R. Nature 2000, 408, 449-453.

(12) Koster, A. J.; Grimm, R.; Typke, D.; Hegerl, R.; Stoschek, A.; Walz, J.; Baumeister, W. J. Struct. Biol. 1997, 120, 276-308.

(13) McIntosh, R.; Nicastro, D.; Mastronarde, D. Trends Cell Biol. 2005, 15, 43-51.

(14) Friedrich, H.; de Jongh, P. E.; Verkleij, A. J.; de Jong, K. P. Chem. Rev. 2009, 109, 1613-1629.

(15) Midgley, P. A.; Ward, E. P. W.; Hungria, A. B.; Thomas, J. M. Chem. Soc. Rev. 2007, 36, 1477-1494.

(16) Ahrenkiel, S. P.; Yu, P. R.; Murphy, J. E.; Nedeljkovic, J. M.; Donohoe, B. S. J. Microsc. (Oxford, U.K.) 2008, 230, 382-387.

(17) Park, J. B.; Lee, J. H.; Choi, H. R. Appl. Phys. Lett. 2007, 90, 093111.

(18) Frank, J. Introduction: Principles of Electron Tomography. In Electron Tomography: Three-Dimensional Imaging with the Transmission Electron Microscope; Frank, J., Ed.; Plenum Press: New York, 1992; p 8.

(19) Deans, S. R. The Radon Transform and Some of Its Applications; John Wiley \& Sons, Inc.: New York, 1983; p 140.

(20) Kremer, J. R.; Mastronarde, D. N.; McIntosh, J. R. J. Struct. Biol. 1996, 116, 71-76.

(21) Frangakis, A. S.; Bohm, J.; Forster, F.; Nickell, S.; Nicastro, D.; Typke, D.; Hegerl, R.; Baumeister, W. Proc. Nat. Acad. Sci. U.S.A. 2002, 99, 14153-14158.

(22) Lebbink, M. N.; Geerts, W. J. C.; van der Krift, T. P.; Bouwhuis, M.; Hertzberger, L. O.; Verkleij, A. J.; Koster, A. J. J. Struct. Biol. 2007, $158,327-335$.

(23) Gommes, C. J.; Friedrich, H.; Wolters, M.; de Jongh, P. E.; de Jong, K. P. Chem. Mater. 2009, 21, 1311-1317.

(24) Gasser, U.; Weeks, E. R.; Schofield, A.; Pusey, P. N.; Weitz, D. A. Science 2001, 292, 258-262.

(25) Schall, P.; Cohen, I.; Weitz, D. A.; Spaepen, F. Nature 2006, 440, 319-323.

(26) ten Wolde, P. R.; Ruiz Montero, M. J.; Frenkel, D. J. Chem. Phys. 1996, 104, 9932-9947.

(27) Eldridge, M. D.; Madden, P. A.; Frenkel, D. Nature 1993, 365, 3537.

(28) Hytch, M. J.; Snoeck, E.; Kilaas, R. Ultramicroscopy 1998, 74, 131146.

(29) Smith, D. K.; Goodfellow, B.; Smilgies, D. M.; Korgel, B. A. J. A. Chem. Soc. 2009, 131, 3281-3290.

(30) Bigioni, T. P.; Lin, X. M.; Nguyen, T. T.; Corwin, E. I.; Witten, T. A.; Jaeger, H. M. Nat. Mater. 2006, 5, 265-270.

(31) Li, W. J.; Fu, T.; He, S. L. Mater. Sci. Eng., A 2006, 441, 239-244.

(32) Klotz, M.; Albouy, P. A.; Ayral, A.; Menager, C.; Grosso, D.; Van der Lee, A.; Cabuil, V.; Babonneau, F.; Guizard, C. Chem. Mater. 2000, 12, 1721-1728.

(33) van Blaaderen, A.; Wiltzius, P. Science 1995, 270, 1177-1179.

(34) Weeks, E. R.; Crocker, J. C.; Levitt, A. C.; Schofield, A.; Weitz, D. A. Science 2000, 287, 627-631.

(35) Kegel, W. K.; van Blaaderen, A. Science 2000, 287, 290-293.

(36) Royall, C. P.; Leunissen, M. E.; van Blaaderen, A. J. Phys., Condens. Matter 2003, 15, S3581-S3596.

\section{NL901212M}

